\title{
Silencing HOXD10 by promoter region hypermethylation activates ERK signaling in hepatocellular carcinoma
}

Yulin Guo ${ }^{1,2 \dagger}$, Yaojun Peng ${ }^{1 \dagger}$, Dan Gao ${ }^{1,3}$, Meiying Zhang ${ }^{1,3}$, Weili Yang ${ }^{1,3}$, Enqiang Linghu', James G. Herman ${ }^{1}$, François Fuks ${ }^{5}$, Guanglong Dong ${ }^{2^{*}}$ and Mingzhou Guo ${ }^{1 *}$ (D)

\begin{abstract}
Background: Hepatocellular carcinoma is the fifth most common malignancy and the third leading cause of cancer-related death worldwide. Dysregulation of HomeoboxD10 (HOXD10) was found to suppress or promote cancer progression in different cancer types. The function and regulation of HOXD10 remain unclear in human hepatocellular carcinoma (HCC).

Methods: Primary HCC samples (117), normal liver tissue samples (15), and 13 HCC cell lines (SNU182, SNU449, HBXF344, SMMC7721, Huh7, HepG2, LM3, PLC/PRF/5, BEL7402, SNU387, SNU475, QGY7703, and Huh1) were included in this study. Methylation-specific PCR, flow cytometry, western blot, transwell, siRNA, and chromatin immunoprecipitation assays were employed.

Results: HOXD10 was methylated in 76.9\% (90/117) of human primary HCC samples. HOXD10 methylation was significantly associated with vessel cancerous embolus, tumor cell differentiation, and the 3-year overall survival rate (all $P<0.05$ ). The expression of HOXD10 was regulated by promoter region methylation. HOXD10 suppressed colony formation, cell proliferation, cell invasion and migration, and induced G2/M phase arrest and apoptosis in HCC cells. HOXD10 suppressed HCC cell xenograft growth in mice. HOXD10 suppresses HCC growth by inhibiting ERK signaling.

Conclusion: HOXD10 is frequently methylated in human HCC, and the expression of HOXD10 is regulated by promoter region methylation. HOXD10 suppresses HCC cell growth both in vitro and in vivo. HOXD10 suppresses human HCC by inhibiting ERK signaling.
\end{abstract}

Keywords: HOXD10, DNA methylation, Hepatocellular carcinoma, IGFBP3, ERK1/2

\section{Background}

Hepatocellular carcinoma (HCC) is the fifth most common malignancy and the third leading cause of cancer-related death worldwide [1]. In China, HCC is the fourth most commonly diagnosed cancer in men, and it is the third leading cause of cancer death for both men and women [2]. The 5-year survival rate remains below $12 \%$ [3]. The mechanisms underlying the development and

\footnotetext{
* Correspondence: gldong@163.com; mzguo@hotmail.com

tEqual contributors

2Department of General Surgery, Chinese PLA General Hospital, \#28 Fuxing Road, Beijing 100853, China

'Department of Gastroenterology and Hepatology, Chinese PLA General Hospital, \#28 Fuxing Road, Beijing 100853, China

Full list of author information is available at the end of the article
}

progression of HCC remain unclear. Chromosomal amplifications $(1 q, 6 p, 8 q, 17 q$, and $20 q)$ and deletions $(4 q, 8 p$, $11 q, 13 q, 16 q$, and $17 p)$ are frequent events in HCC [4]. In $5-10 \%$ of HCC patients, high-level amplifications have been described in $6 p 21$ (vascular endothelial growth factor A, VEGFA) and 11q13 (cyclin D1, CCND1) [5]. Accumulating evidence has shown that epigenetic as well as genetic alterations play important roles in the development of many cancers [6-8]. Epigenetic inactivation of tumor suppressor genes has been frequently found in HCC [9]. Homeobox (HOX) genes encode homeoproteins, which share a common homeodomain and serve as important transcription factors targeting downstream proteins [10]. Homeoproteins play an important role in 
development and carcinogenesis by modulating cell growth, migration, cell cycle, and apoptosis [11-14]. Homeobox D10 (HOXD10) is a member of the homeobox gene family. HOXD10 expression levels and functions vary by cancer type [14-17]. In this study, we analyzed the regulation and the function of HOXD10 in human HCC.

\section{Methods}

\section{Human tissue samples and cell lines}

Primary human hepatocellular carcinoma samples (117 cases) and normal liver tissue samples (15 cases) were collected from the Chinese PLA General Hospital in Beijing between 1 July 2010 and 1 January 2014. The median age of the cancer patients was 55 years old (range 29-71), and the ratio of males/females was 6.3:1. All cancer samples were classified according to TNM staging (AJCC 2010). Forty cases of available matched cancer adjacent tissue paraffin samples were included in this study. All samples were collected following the guidelines approved by the Institutional Review Boards of the Chinese PLA General Hospital with written informed consent from patients. HCC cell lines, which included SNU182, SNU387, HBXF344, SNU475, HepG2, PLC/PRF/5, Huh7, BEL7402, LM3, SNU449, SMMC7721, QGY7703, and Huh1 were previously established from human primary HCC [18]. All cells were maintained in 90\% RPMI 1640 (Invitrogen, Carlsbad, CA) supplemented with $10 \%$ fetal bovine serum.

\section{5-Aza-2'-deoxycytidine treatment}

HCC cell lines were split to a low density (30\% confluence) $12 \mathrm{~h}$ before treatment. Cells were treated with 5 -aza-2'deoxycytidine (5-aza) (Sigma, St. Louis, MO) at a concentration of $2 \mu \mathrm{M}$. Growth medium conditioned with 5-aza at a concentration of $2 \mu \mathrm{M}$ was exchanged every $24 \mathrm{~h}$ for a total of $96 \mathrm{~h}$ of treatment.

\section{RNA isolation and semi-quantitative RT-PCR}

Total RNA was isolated by Trizol reagent (Life Technologies, Gaithersburg, MD). First-strand cDNA was synthesized according to the manufacturer's instructions (Invitrogen, Carlsbad, CA). PCR primers for HOXD10 are listed in Additional file 1: Table S1. The primer sets for HOXD10 were designed to span intronic sequences between adjacent exons in order to control for genomic DNA contamination. RT-PCR was amplified for 33 cycles. GAPDH was amplified for 25 cycles as an internal control.

\section{Bisulfite modification, methylation-specific PCR (MSP), and bisulfite sequencing}

DNA was prepared by the proteinase $\mathrm{K}$ method. Bisulfite treatment was carried out as previously described [19]. MSP primers were designed according to genomic sequences around transcription start sites (TSS) and synthesized to detect unmethylated (U) and methylated (M) alleles. Bisulfite sequencing (BSSQ) was performed as previously described [20]. BSSQ products were amplified by primers flanking the targeted regions including MSP products. All primers are listed in Additional file 1: Table S1.

\section{Immunohistochemistry}

Immunohistochemistry (IHC) was performed in HCC tissue samples and paired adjacent tissue samples. The HOXD10 antibody was diluted to 1:100 (Abcam, Cambridge, UK). The staining intensity and extent of the staining area were scored using the German semi-quantitative scoring system as previously described $[20,21]$.

\section{Construction of HOXD10 expression vector and transfection assay}

Full-length HOXD10 cDNA (GenBank accession number NM_002148.3) was cloned into the pcDNA3.1 expression vector. Transient transfection was performed using Lipofectamine 3000 (Intrivogen, Carlsbad, CA) according to the manufacturer's instructions.

\section{Cell viability detection}

Cells were plated into 96-well plates at a density of $2 \times 10^{3}$ cells/well, and cell viability was measured by the 3-(4,5-dimethylthiazol-2-yl)-2,5-diphenyltetrazolium bromide (MTT) assay (KeyGEN Biotech, Nanjing, China) at 0, 24, 48, and $72 \mathrm{~h}$. Absorbance was measured using a microplate reader (Thermo Multiskan MK3, MA, USA) at a wavelength of $490 \mathrm{~nm}$.

\section{Colony formation assay}

Cells were seeded into 6-well culture plates at a density of 800 cells per well in triplicate and cultured for 2 weeks. For Huh7 and SMMC7721 cells, growth medium was conditioned with G418 (Invitrogen, Carlsbad, CA) at 300 and $50 \mu \mathrm{g} / \mathrm{ml}$, respectively, and was exchanged every $24 \mathrm{~h}$. Cells were then fixed with $75 \%$ ethanol for $30 \mathrm{~min}$, stained with $0.2 \%$ crystal violet (Beyotime, Nanjing, China) for 20 min and counted.

\section{Flow cytometry}

For cell cycle analysis, cells were serum starved $12 \mathrm{~h}$ for synchronization and then re-stimulated with $10 \%$ FBS for $24 \mathrm{~h}$. Cells were fixed with $70 \%$ ethanol and prepared for cell cycle detection using the Cell Cycle Detection Kit (KeyGen Biotech, Nanjing, China). Cells were then sorted by a FACSCalibur (BD Biosciences, San Jose, CA) and analyzed by the Modfit software (Verity Software House, ME, USA). For apoptosis analysis, the Annexin VFITC/PI Apoptosis Detection Kit (KeyGen Biotechnology, 
China) was used according to manufacturer's instructions. Each sample was analyzed by flow cytometry with a FACScan Flow Cytometer (Becton-Dickinson Biosciences, Mansfield, MA).

\section{Transwell assay}

Cells were suspended in serum-free medium. Cells $\left(2 \times 10^{4}\right)$ were placed into the upper chamber of an 8$\mu \mathrm{m}$ pore size transwell apparatus (Corning, NY, USA) and incubated for $24 \mathrm{~h}$. Cells that migrated to the lower surface of the membrane were stained with crystal violet and counted in three independent high-power fields $(\times 200)$. For invasion analysis, cells $\left(3 \times 10^{4}\right)$ were seeded into the upper chamber of a transwell apparatus coated with Matrigel (BD Biosciences, San Jose, CA) and incubated for $48 \mathrm{~h}$. Cells that invaded into the lower membrane surface were stained with crystal violet and counted in three independent high-power fields $(\times 200)$.

\section{Chromatin immunoprecipitation}

Chromatin immunoprecipitation (ChIP) was performed in HOXD10 highly expressed Huh1 cells using HOXD10 monoclonal antibody (Life Span Bio Sciences, Inc., WA, USA) or normal rabbit IgG (negative control) according to the EpiTect ChIP One Day Kit (Qiagen, Hilden, Germany). Two primers encompassing HOXD10 binding sites in different regions of the IGFBP3 promoter were designed as shown in Additional file 1: Table S1.

\section{SiRNA knockdown technique}

SiRNAs targeting HOXD10 and the RNAi negative control duplex were used in this study. The sequences of the siRNAs are listed in Additional file 1: Table S1 (Gene Pharma Co, Shanghai, China). The RNAi oligonucleotide and RNAi negative control duplex were transfected into HOXD10 highly expressing QGY7703 and Huh1 cells.

\section{Western blot}

Proteins from HCC cells were collected $48 \mathrm{~h}$ after transfection. For extracellular signal-regulated kinase (ERK) signaling analysis, cells were starved with serumfree medium for $24 \mathrm{~h}$ after transfection. These cells were then stimulated with medium containing $10 \%$ serum for 15 to $60 \mathrm{~min}$ before collection. Western blot was performed as described previously [21]. Antibodies were diluted according to manufacturer's instructions. The primary antibodies were as follows: HOXD10 (Life Span Bio Sciences, Inc., WA, USA), IGFBP3 (Protein Tech Group, Chicago, IL, USA), ERK1/2 (Bioworld Tech, MN, USA), p-ERK1/2 (Bioworld Tech, MN, USA), MMP2 (Protein Tech Group, Chicago, IL, USA), MMP9 (Protein Tech Group, Chicago, IL, USA), cyclinB1 (Protein Tech Group, Chicago, IL, USA), cdc-2 (Protein Tech Group, Chicago, IL, USA), bcl-2 (Protein Tech Group, Chicago,
IL, USA), cleaved caspase 3 (Protein Tech Group, Chicago, IL, USA), and $\beta$-actin (Bioworld Tech, MN, USA).

\section{HOXD10 unexpressed and re-expressed SMMC7721 cell xenograft mouse model}

Stably transfected SMMC7721 cell line with pLenti6 vector or pLenti6-HOXD10 vector $\left(6 \times 10^{6}\right.$ cells diluted in phosphate-buffered saline and matrigel mixed at the ratio of 1:1) were injected subcutaneously into the dorsal right side of 4-week-old female Balb/c nude mice. Each group includes six mice. Tumor volume was measured every 3 days. Tumor volume was calculated according to the formula: $V=L \times W 2 / 2$, where $V$ represents volume (mm3), $L$ represents biggest diameter $(\mathrm{mm})$, and $W$ represents smallest diameter $(\mathrm{mm})$. Mice were sacrificed on the 39th day after inoculation and tumor was weighted. All procedures were approved by the Animal Ethics Committee of the Chinese PLA General Hospital.

\section{Statistical analysis}

SPSS 17.0 software (IBM, NY, USA) was used for data analysis. Categorical variables were analyzed using the chi-squared test or Fisher's exact test. The two-tailed independent sample $t$ test was applied to determine the statistical significance of the differences between the two experimental groups. For matched HCC and adjacent tissue samples, paired Student's $t$ test was employed. Survival rates were calculated by the Kaplan-Meier method, and differences in survival curves were evaluated using the log-rank test. Cox proportional hazards models were fit to determine independent associations of HOXD10 methylation with 3-year OS outcomes. Two-sided tests were used to determine the significance, and $P<0.05$ was considered as statistically significant.

\section{Results \\ HOXD10 is silenced by promoter region hypermethylation in HCC cells}

Semi-quantitative RT-PCR was employed to detect the expression of HOXD10 in HCC cells. Loss of HOXD10 expression was found in SNU182, SNU449, HBXF344, SMMC7721, Huh7, HepG2, LM3, PLC/PRF/5, and BEL7402 cells. Reduced expression of HOXD10 was found in SNU387 and SNU475 cells. HOXD10 was highly expressed in QGY7703 and Huh1 cells (Fig. 1a). MSP was employed to detect promoter region methylation. MSP primers were designed around the transcription start site in the CPG islands within the HOXD10 gene promoter region. Complete methylation was found in SNU182, SNU449, HBXF344, SMMC7721, Huh7, HepG2, LM3, PLC/PRF/5, and BEL7402 cells, partial methylation was found in SNU387 and SNU475 cells, and unmethylation was found in QGY7703 and Huh1 


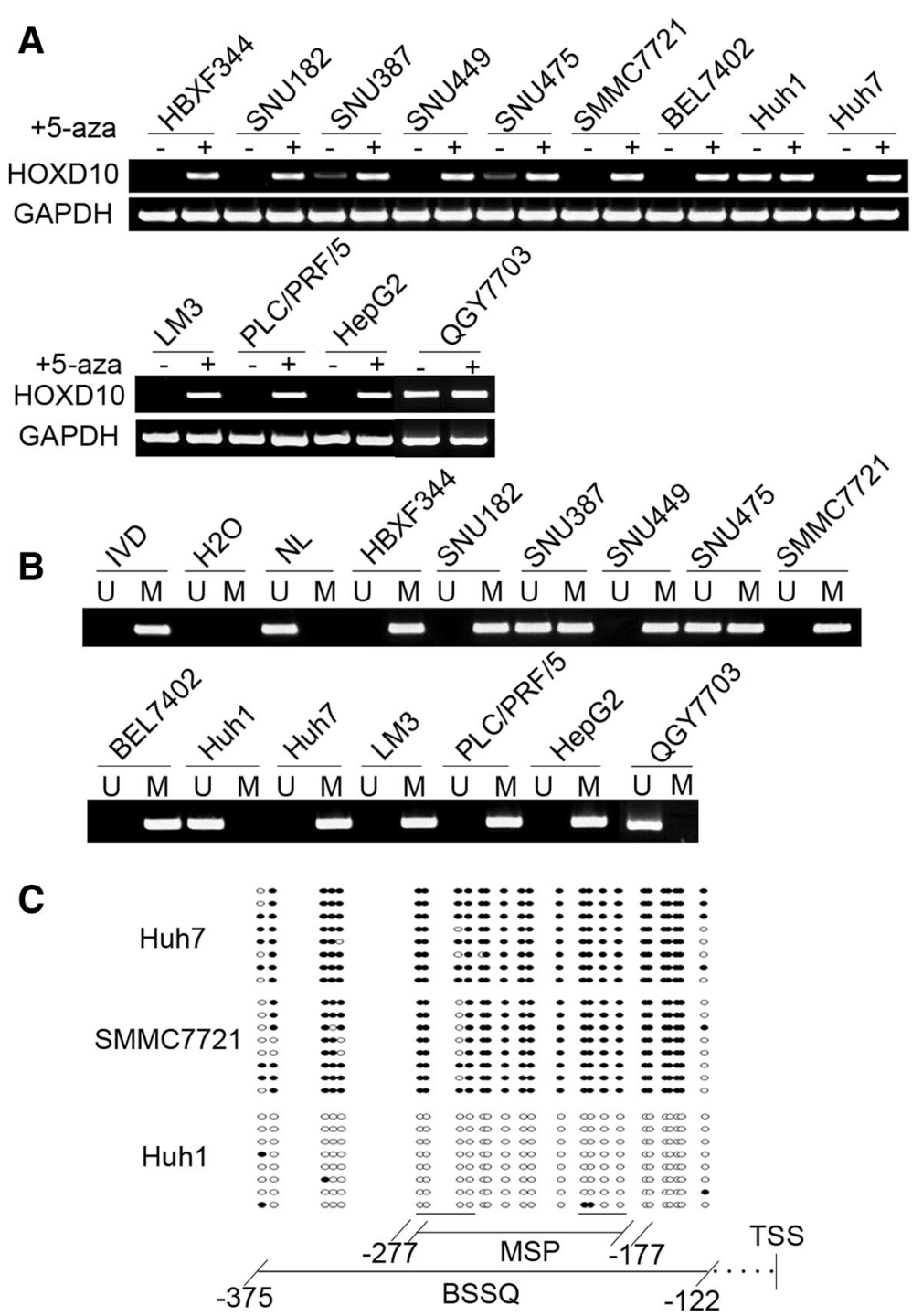

Fig. 1 HOXD10 expression and methylation status in human HCC cells. a Semi-quantitative RT-PCR shows HOXD10 expression levels in HCC cell lines. HBXF344, SNU182, SNU387, SNU449, SNU475, SMMC7721, BEL7402, Huh1, Huh7, LM3, PLC/PRF/5, HepG2, and QGY7703 are HCC cell lines. 5-AZA: 5-aza-2'-deoxycytidine; GAPDH: internal control of RT-PCR; (-): absence of 5-AZA; (+): presence of 5-AZA. b MSP results of HOXD10 in HCC cell lines. U: unmethylated alleles; M: methylated alleles; IVD: in vitro methylated DNA, serves as methylation control; NL: normal peripheral lymphocytes DNA, serves as unmethylated control; $\mathrm{H}_{2} \mathrm{O}$ : double distilled water. c BSSQ results of HOXD10 in SMMC7721, Huh7 and Huh1 cells. Double-headed arrow: MSP PCR product size was $100 \mathrm{bp}$ and bisulfite sequencing focused on a $254 \mathrm{bp}$ region of the CpG island (from - 375 to - 122) around the HOXD10 transcription start site. Filled circles: methylated CpG sites, open circles: unmethylated CpG sites. TSS: transcription start site

cells (Fig. 1b). These results demonstrate that loss or reduced expression of HOXD10 is correlated with promoter region methylation.

To further validate that the expression of HOXD10 was regulated by promoter region methylation, HCC cells were treated with the DNA methylation transferase inhibitor, 5-aza-2'-deoxycytidine (5-aza). Re-expression of HOXD10 was found in SNU182, SNU449, HBXF344, SMMC7721, Huh7, HepG2, LM3, PLC/PRF/5, and BEL7402 cells, and increased expression of HOXD10 was found in SNU387 and SNU475 cells. No expression change was found in QGY7703 and Huh1 cells (Fig. 1a). These results suggest that HOXD10 expression is regulated by promoter region methylation in HCC cells. To further validate the MSP results and examine the methylation density in the promoter region, BSSQ was performed in SMMC7721, Huh7, and Huh1 cells. BSSQ results were consistent with the MSP results, showing dense promoter region methylation in SMMC7721 and Huh7 cells and no methylation in Huh1 cells (Fig. 1c). 
HOXD10 is frequently methylated in human primary HCC, and reduced expression of HOXD10 is associated with promoter region hypermethylation

To determine the methylation status of HOXD10 in human primary HCC, 117 cases of primary HCC and 15 cases of normal liver tissue samples were examined by MSP. HOXD10 was methylated in 76.9\% (90/117) of primary HCC samples, but no methylation was detected in normal liver tissue samples (Fig. 2a). As shown in Table 1, methylation of HOXD10 was more frequently in patients with vessel cancerous embolus $(p<0.05)$ and poorly differentiated tumors $(P<0.05)$, but no association was found between HOXD10 methylation and age, gender, HBV infection, cirrhosis, tumor size, number of lesions, TNM stage, and lymph node metastasis (all
$P>0.05)$.The median follow-up period for patients was 23 months (range, 0-76 months).Four patients in the unmethylated group and 17 patients in the methylated group were lost to follow-up. Kaplan-Meier plots indicated that methylation of HOXD10 was associated with poor 3-year overall survival $(\mathrm{OS})(P=0.048$, Fig. 2b). While, according to Cox proportional hazards model analysis, HOXD10 methylation was not an independent prognostic factor for 3-year OS after adjusting for tumor differentiation, vessel cancerous embolus, and TNM stage $(P=0.127$, Table 2$)$.

The expression of HOXD10 was evaluated by immunohistochemistry (IHC) in 40 cases of available matched primary HCC and adjacent tissue samples. Staining of HOXD10 was mainly localized in the nucleus, and its

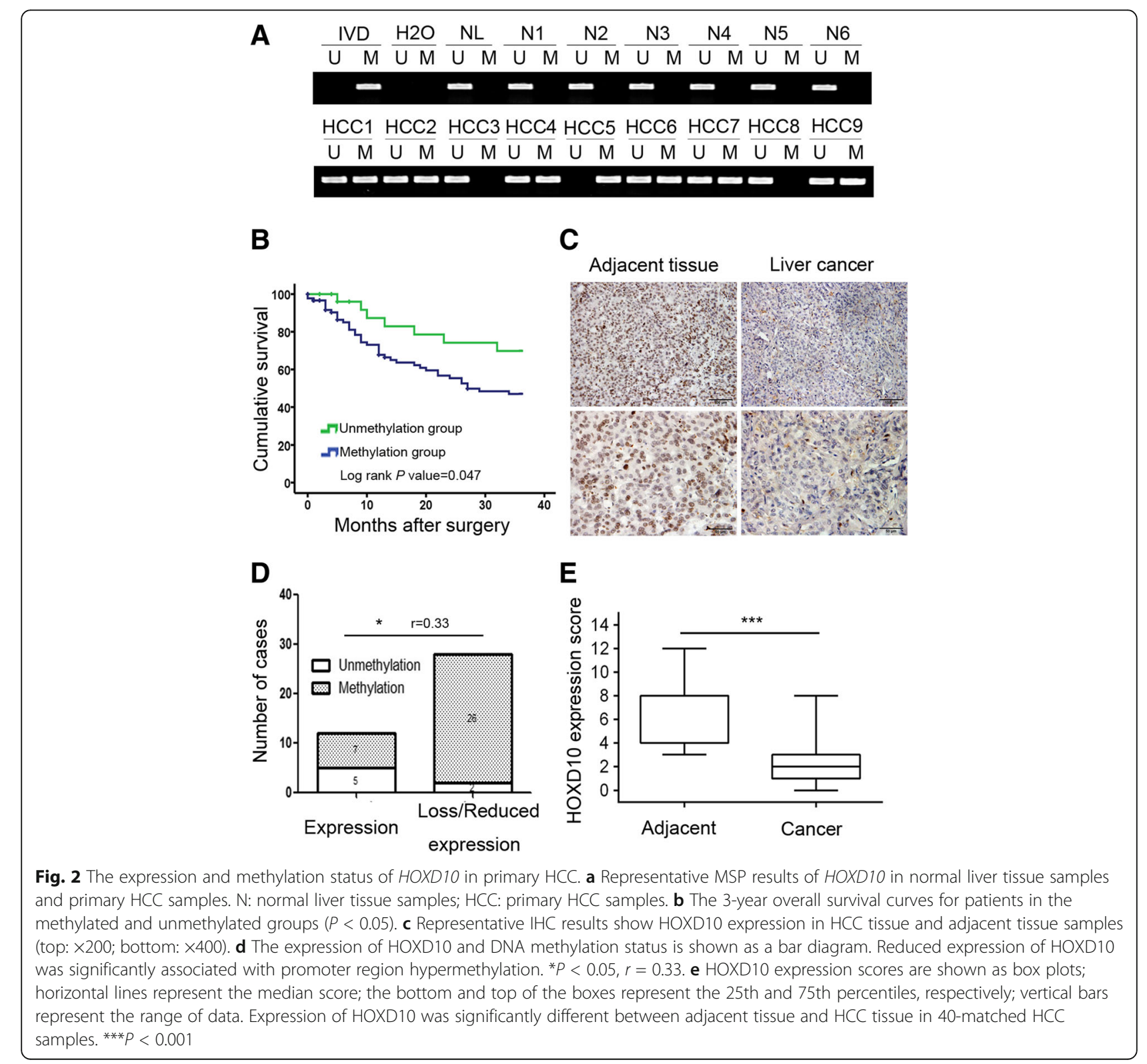


Table 1 Clinical factors and HOXD10 methylation in 117 cases of HCC samples

\begin{tabular}{|c|c|c|c|c|}
\hline \multirow[t]{2}{*}{ Clinical factor } & \multirow[t]{2}{*}{ No. } & \multicolumn{2}{|l|}{ HOXD10 methylation status } & \multirow[t]{2}{*}{${ }^{*} P$ value } \\
\hline & & Unmethylated $n=27$ (23.1\%) & Methylated $n=90(76.9 \%)$ & \\
\hline Age (year) & & & & 0.799 \\
\hline$<60$ & 80 & 19 & 61 & \\
\hline$\geq 60$ & 37 & 8 & 29 & \\
\hline Gender & & & & 0.248 \\
\hline Male & 101 & 21 & 80 & \\
\hline Female & 16 & 6 & 10 & \\
\hline HBV infection & & & & 0.762 \\
\hline Yes & 85 & 19 & 66 & \\
\hline No & 32 & 8 & 24 & \\
\hline Liver cirrhosis & & & & 0.093 \\
\hline Yes & 88 & 17 & 71 & \\
\hline No & 29 & 10 & 19 & \\
\hline Tumor size $(\mathrm{cm})$ & & & & 0.231 \\
\hline$\leq 5$ & 37 & 6 & 31 & \\
\hline$>5$ & 80 & 21 & 59 & \\
\hline Number of lesions & & & & 0.681 \\
\hline 1 & 92 & 22 & 70 & \\
\hline$\geq 1$ & 25 & 5 & 20 & \\
\hline Differentiation & & & & $0.044^{*}$ \\
\hline Well & 8 & 4 & 4 & \\
\hline Moderate & 74 & 19 & 55 & \\
\hline Poor & 35 & 4 & 31 & \\
\hline TNM stage & & & & 0.377 \\
\hline Stage I + stage I| & 52 & 14 & 38 & \\
\hline Stage III + stage IV & 65 & 13 & 52 & \\
\hline Lymph node metastasis & & & & 0.867 \\
\hline Negative & 112 & 26 & 86 & \\
\hline Positive & 5 & 1 & 4 & \\
\hline Vessel cancerous embolus & & & & $0.049^{*}$ \\
\hline Negative & 87 & 24 & 63 & \\
\hline Positive & 30 & 3 & 27 & \\
\hline
\end{tabular}

${ }^{*} P$ values are obtained from chi-square test, significant difference, ${ }^{*} P<0.05$

expression was significantly reduced in primary $\mathrm{HCC}$ compared to adjacent tissue samples $(P<0.001$, Fig. $2 \mathrm{c}, \mathrm{e})$. In 40 cases of available primary $\mathrm{HCC}$, loss or reduced expression of HOXD10 was found in 28 cases. Of these 28 case samples, 26 cases were methylated and 2 cases were unmethylated. Loss or reduced expression of HOXD10 was significantly associated with promoter region hypermethylation $(P<0.05, r=0.33$, Fig. 2d). These results indicate that HOXD10 expression is regulated by promoter region methylation in primary $\mathrm{HCC}$.

\section{HOXD10 suppresses proliferation of HCC cells}

To evaluate the effects of HOXD10 on HCC cell proliferation, cell viability was determined by the MTT assay. The OD value was $0.63 \pm 0.05$ vs. $0.50 \pm 0.05(P<0.01)$ and $0.68 \pm 0.01$ vs. $0.53 \pm 0.02(P<0.05)$ before and after restoration of HOXD10 expression in HOXD10 unexpressed SMMC7721 and Huh7 cells, respectively. The OD value was reduced significantly (Fig. 3a). In HOXD10 highly expressed QGY7703 cells, the OD value was $0.45 \pm 0.03$ vs. $0.50 \pm 0.04$ before and after knockdown of HOXD10 expression. The OD value was 
Table 2 Univariate and multivariate analysis of HOXD10 methylation status with 3-year overall survival (OS) in HCC patients

\begin{tabular}{|c|c|c|c|c|}
\hline \multirow[t]{3}{*}{ Clinical factor } & \multicolumn{4}{|l|}{ 3-year OS } \\
\hline & \multicolumn{2}{|l|}{ Univariate analysis } & \multicolumn{2}{|l|}{ Multivariate analysis } \\
\hline & $\mathrm{HR}(95 \% \mathrm{Cl})$ & $P$ value & $\mathrm{HR}(95 \% \mathrm{Cl})$ & $P$ value \\
\hline Age (<60 vs. $\geq 60$ years) & $1.306(0.775-2.201)$ & 0.317 & & \\
\hline Gender (male vs. female) & $0.717(0.376-1.368)$ & 0.312 & & \\
\hline HOXD10 (methylation vs. unmethylation) & $1.920(1.006-3.664)$ & $0.048^{*}$ & $1.676(0.864-3.252)$ & 0.127 \\
\hline HBV infection (yes vs. no) & $0.664(0.374-1.178)$ & 0.161 & & \\
\hline Liver cirrhosis (yes vs. no) & $0.888(0.513-1.538)$ & 0.672 & & \\
\hline Tumor size ( $\leq 5$ vs. $>5 \mathrm{~cm})$ & $0.707(0.412-1.212)$ & 0.207 & & \\
\hline Number of lesions ( 1 vs. $\geq 1$ ) & $0.770(0.439-1.349)$ & 0.361 & & \\
\hline Differentiation (well or moderate vs. poor) & $0.787(0.476-1.301)$ & 0.351 & $1.267(0.740-2.172)$ & 0.388 \\
\hline TNM stage (stage I + stage II vs. stage III + stage IV) & $0.391(0.233-0.656)$ & $0.000^{* * *}$ & $0.502(0.279-0.902)$ & $0.021^{*}$ \\
\hline Lymph node metastasis (negative vs. positive) & $0.431(0.172-1.075)$ & 0.071 & & \\
\hline Vessel cancerous embolus (negative vs. positive) & $0.356(0.217-0.584)$ & $0.000^{* * *}$ & $0.503(0.287-0.884)$ & $0.017^{*}$ \\
\hline
\end{tabular}

${ }^{*} P<0.05,{ }^{* * *} P<0.001$

increased significantly $(P<0.05$, Fig. 3a). These results suggest that HOXD10 suppresses HCC cell viability. Colony formation assays were performed to evaluate the effect of HOXD10 on clonogenicity. As shown in Fig. 3b, the colony number was $155.67 \pm 14.64$ vs. $61.0 \pm 7.55$ in SMMC7721 cells $(P<0.01)$ and $139.67 \pm 15.18$ vs. $97.33 \pm 10.07$ in Huh7 cells $(P<0.05)$ before and after re-expression of HOXD10, showing significant reduction in colony formation with HOXD10 re-expression. In HOXD10 highly expressed QGY7703 cells, the colony number was $51.67 \pm 6.43$ vs. $91.67 \pm 20.50$ before and knockdown of HOXD10. The colony number was significantly increased after knockdown of HOXD10 expression $(P<0.05$, Fig. 3b). These results demonstrate that HOXD10 suppresses HCC cell growth.

\section{HOXD10 induces cell apoptosis}

To explore the role of HOXD10 in apoptosis, flow cytometry was performed. The percentages of apoptotic cells were $9.30 \pm 0.15 \%$ vs. $11.00 \pm 0.57 \%$ in SMMC7721 cells $(P<0.05)$ and $4.86 \pm 0.26 \%$ vs. $6.57 \pm 0.55 \%$ in Huh7 cells $(P<0.05)$ before and after re-expression of HOXD10. The percentage of apoptotic cells was increased significantly (Fig. 3c) after re-expression of HOXD10. In HOXD10 highly expressed QGY7703 cells, the percentage of apoptotic cells was $13.56 \pm 2.03 \%$ before knockdown of HOXD10 and $9.83 \pm 0.36 \%$ after knockdown of HOXD10. The percentage of apoptotic cells was reduced significantly $(P<0.05$, Fig. $3 \mathrm{c})$. To further validate HOXD10-induced apoptosis in HCC cells, cleaved capase- 3 and bcl-2 levels were examined by western blot. The levels of cleaved capase- 3 were increased and the levels of bcl- 2 were reduced after reexpression of HOXD10 in SMMC7721 and Huh7 cell. In HOXD10 highly expressed QGY7703 cells, the levels of cleaved capase- 3 were reduced and the levels of bcl-2 were increased after knockdown of HOXD10 (Fig. 3d). These results suggest that HOXD10 induces apoptosis in HCC cells.

\section{HOXD10 induces G2/M phase arrest}

The role of HOXD10 in the cell cycle was analyzed by flow cytometry. As shown in Fig. 4a, the distribution of cell phase in HOXD10 unexpressed and re-expressed SMMC7721 cells was $61.35 \pm 1.75 \%$ vs. $56.86 \pm 0.69 \%$ in G0/G1 phase $(P<0.05), 29.55 \pm 0.59 \%$ vs. $27.04 \pm 1.43 \%$ in $S$ phase, and $9.10 \pm 1.76 \%$ vs. $16.09 \pm 0.80 \%$ in $\mathrm{G} 2 / \mathrm{M}$ phase $(P<0.05)$. In Huh7 cells, the cell phase distribution was $63.95 \pm 0.92 \%$ vs. $52.27 \pm 0.23 \%$ in $\mathrm{G0} / \mathrm{G} 1$ phase $(P<0.01), 24.85 \pm 1.09 \%$ vs. $26.16 \pm 0.38 \%$ in $S$ phase, and $11.20 \pm 0.36 \%$ vs. $21.58 \pm 0.15 \%$ in $\mathrm{G} 2 / \mathrm{M}$ phase $(P<0.01)$ before and after restoration of HOXD10 expression (Fig. 4a). G2/M phase was increased significantly after re-expression of HOXD10 in HCC cells. To further validate these results, siRNA knockdown technique was employed. The cell phase distribution was $53.00 \pm 0.61 \%$ vs. $61.28 \pm 0.38 \%$ in $\mathrm{G} 0 / \mathrm{G} 1$ phase $(P<0.01), 27.30 \pm 0.52 \%$ vs. $26.40 \pm 0.40 \%$ in $S$ phase, and $19.67 \pm 0.12 \%$ vs. $12.20 \pm 0.61 \%$ in $\mathrm{G} 2 / \mathrm{M}$ phase before and after knockdown of HOXD10 in HOXD10 highly expressed QGY7703 cells (Fig. 4a). The percentage of $\mathrm{G} 2 / \mathrm{M}$ phase was reduced significantly after knockdown of HOXD10 $(P<0.01)$. These results suggest that HOXD10 induces G2/M phase arrest in HCC cells.

The induction of G2/M checkpoint arrest by HOXD10 was further validated by detecting $\mathrm{G} 2 / \mathrm{M}$ phase-related proteins. The expression levels of cyclinB1 and cdc-2 were dramatically reduced after re-expression of HOXD10 in SMMC7721 and Huh7 cells, and the levels of cyclinB1 and cdc-2 expression were increased obviously after 


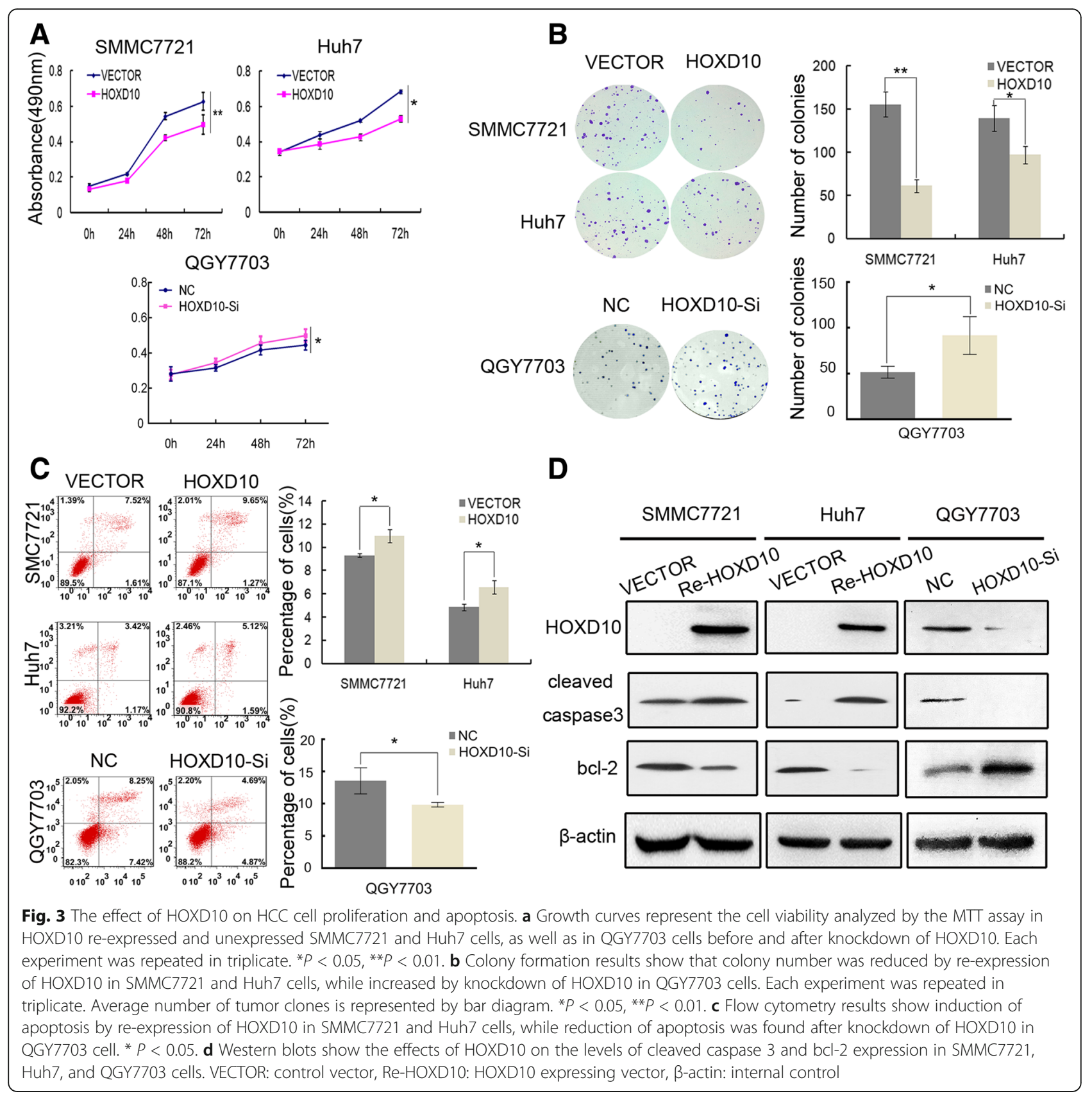

knockdown of HOXD10 in HOXD10 highly expressed QGY7703 cells (Fig. 4b). Above results suggest that HOXD10 inhibits cell proliferation in HCC.

\section{HOXD10 suppresses cell invasion and migration in HCC}

The transwell assay was employed to evaluate the effects of HOXD10 on cell invasion. The number of cells for each high-power field under the microscope was $140.00 \pm 10.00$ vs. $70.33 \pm 11.68$ in SMMC7721 cells and $159.00 \pm 14.73$ vs. $93.33 \pm 7.51$ in Huh7 cells before and after restoration of HOXD10 expression. The number of invasive cells was reduced significantly after re-expression of HOXD10 in SMMC7721 and Huh7 cells (all $P<0.001$, Fig. 5a). In HOXD10 highly expressed QGY7703 cells, the number of invasive cells for each high-power field under the microscope was $78.00 \pm 6.08$ vs $129.30 \pm 10.07$ before and after knockdown of HOXD10. The number of invasive cells was increased significantly $(P<0.01$, Fig. $5 \mathrm{a})$. To explore the mechanism of HOXD10 in HCC cell invasion, MMP2 and MMP9 were examined by western blot. The expression levels of MMP2 and MMP9 were reduced after re-expression of HOXD10 in SMMC7721 and Huh7 cells. While, the expression levels of MMP2 and MMP9 was increased obviously after knockdown of HOXD10 in 

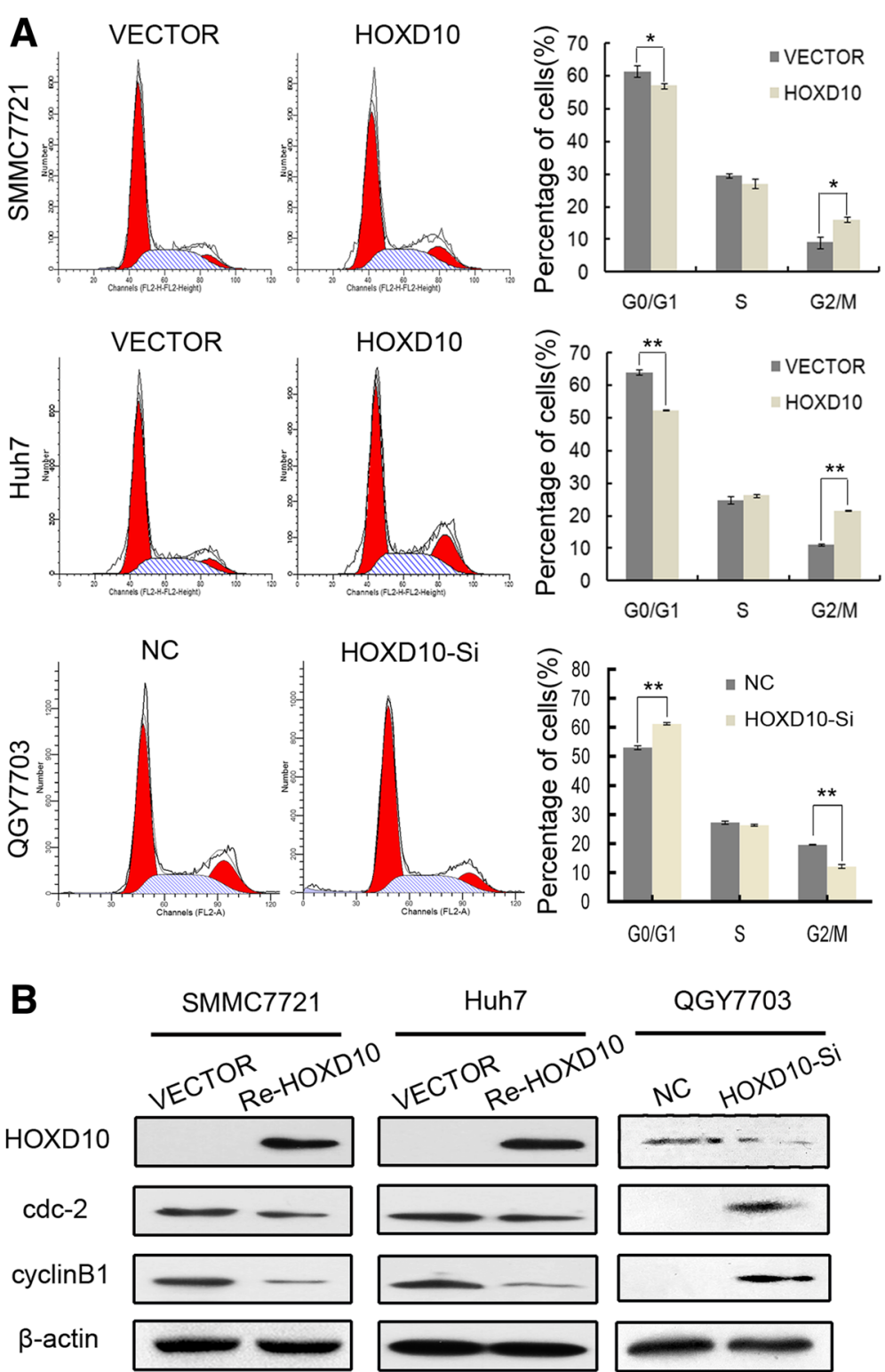

Huh7
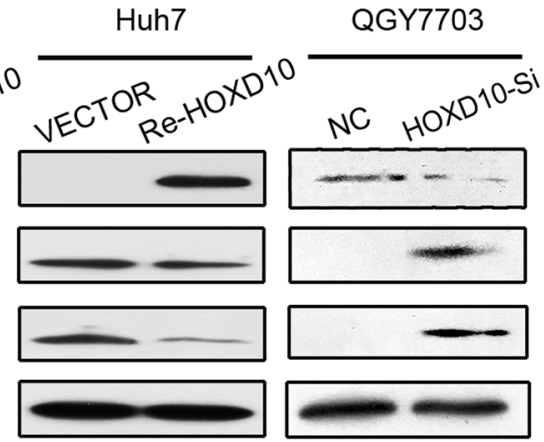

Fig. 4 The effect of HOXD10 on HCC cell cycle. a Cell phase distribution in HOXD10 unexpressed and re-expressed SMMC7721 and Huh7 cells, as well as in QGY7703 cells before and after knockdown of HOXD10. The ratio is presented by bar diagram. Each experiment was repeated three times. ${ }^{*} P<0.05$, ${ }^{*} P<0.01$. b The expression of HOXD10, cyclinB1, and cdc-2 was detected by western blot in HOXD10 unexpressed and re-expressed SMMC7721 and Huh7 cells, as well as in QGY7703 cells before and after knockdown of HOXD10. $\beta$-actin: internal control

HOXD10 highly expressed QGY7703 cells (Fig. 5c). The results suggest that HOXD10 inhibits cell invasion in HCC cells.

Next, the transwell assay was performed in the absence of extracellular matrix (ECM) gel coating to explore the effects of HOXD10 on cell migration. The numbers of migrated cells for each high-power field under the microscope were $251.00 \pm 11.36$ vs. $197.67 \pm 11.02$ in SMMC7721 cells $(P<0.05)$ and $402.00 \pm 20.52$ vs. $203.00 \pm 12.29(P<0.001)$ in Huh7 cells before and after restoration of HOXD10 expression.
The number of migrated cells was $221.70 \pm 19.76$ vs. $317.00 \pm 24.64$ before and after knockdown of HOXD10 in HOXD10 highly expressed QGY7703 cells $(P<0.01$, Fig. 5b). The results indicate that HOXD10 inhibits cell migration in HCC cells.

\section{HOXD10 inhibits ERK signaling in HCC cells}

HOXD10 has been demonstrated to act as a transcription factor targeting the promoter region of IGFBP3 in gastric cancer $[15,22]$. IGFBP3 may activate different signaling pathways in different cancers [23]. In the NSCLC cell line 


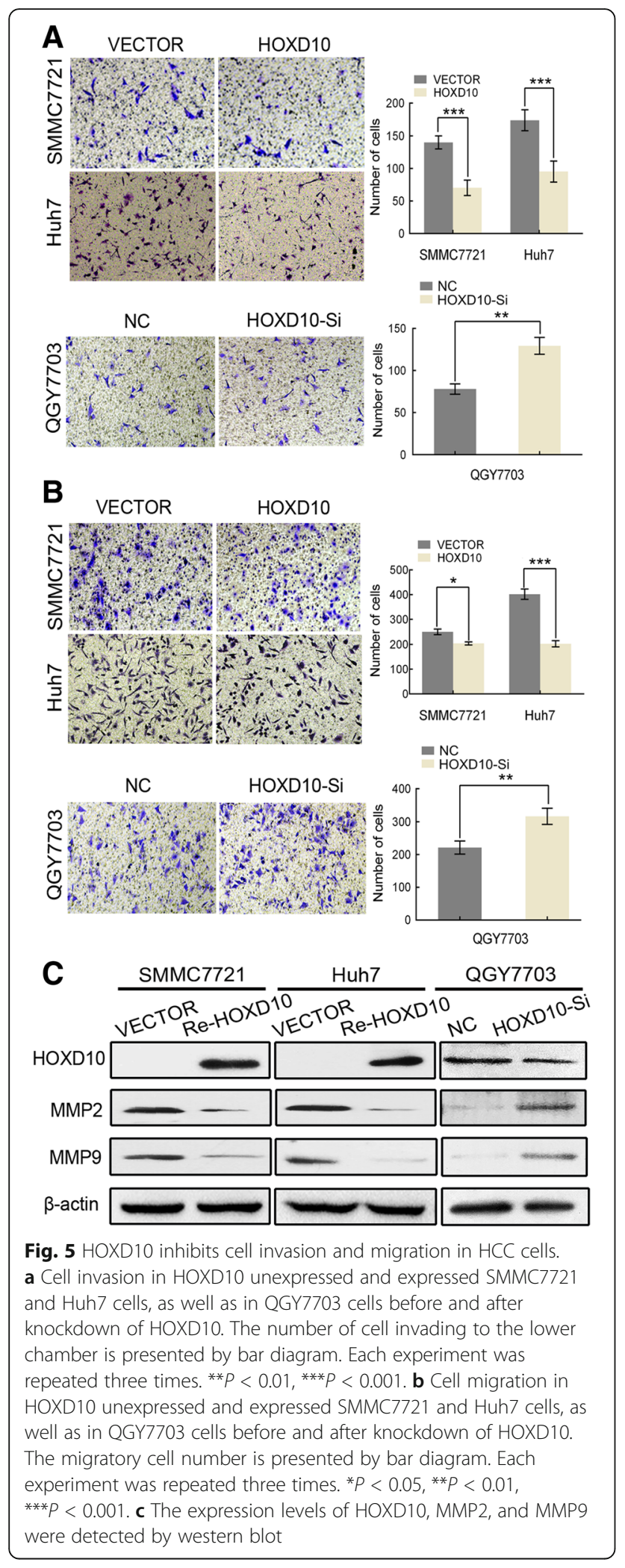

H1299, IGFBP3 interacts with and inactivates ERK1/2 by inhibiting ERK1/2 phosphorylation [24]. In human HCC, the signaling pathway involving HOXD10 remains unclear. To further understand the mechanism of HOXD10 in HCC and determine whether it binds to IGFBP3, ChIP assays were performed. The IGFBP3 promoter region was pulled down by the HOXD10 antibody in HOXD10 highly expressing Huh1 cells (Fig. 6a). Results of the ChIP assay suggest that HOXD10 interacts with the promoter region of IGFBP3. To further analyze the role of HOXD10, the expression levels of IGFBP3 were examined by western blot in HOXD10 unexpressed and re-expressed SMMC7721 and Huh7 cells. The expression of IGFBP3 increased after

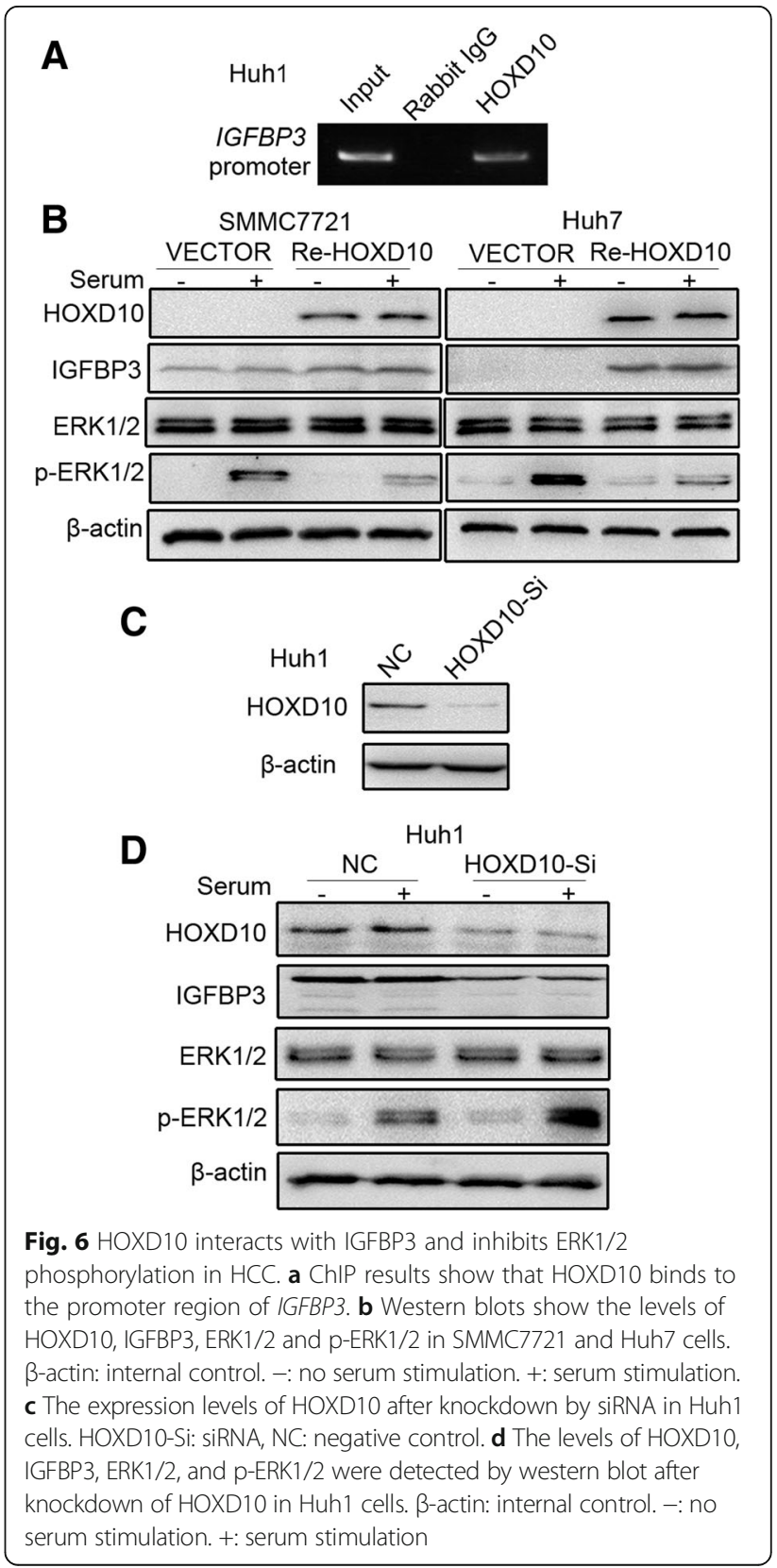


re-expression of HOXD10, suggesting that HOXD10 upregulates IGFBP3 in HCC cells (Fig. 6b).

Next, we detected phosphorylation status of ERK1/2 by western blot to further explore the mechanism of IGFBP3 in regulation of downstream signaling. After $24 \mathrm{~h}$ of serum starvation followed by $10 \%$ serum stimulation for $45 \mathrm{~min}$, the levels of phosphorylated ERK1/2 were reduced in HOXD10 re-expressed SMMC7721 cells compared to HOXD10 unexpressed SMMC7721 cells. Similarly, the levels of phosphorylated ERK1/2 were reduced by HOXD10 in Huh7 cells after 10\% serum stimulation for $30 \mathrm{~min}$. These results indicate that HOXD10 inhibits ERK1/2 phosphorylation by upregulating IGFBP3 expression (Fig. 6b). To further validate the inhibitory effect of HOXD10 on ERK signaling, siRNA knockdown technique was used in HOXD10 highly expressing Huh1 cells. HOXD10-SiR1 effectively knocked down HOXD10 in Huh1 cells (Fig. 6c). The levels of phosphorylated ERK1/2 were increased by knockdown of HOXD10 in 10\% FBS-stimulated Huh1 cells (Fig. 6d). These results suggest that HOXD10 inhibits ERK signaling by upregulating IGFBP3 in HCC.

\section{HOXD10 suppresses human HCC cell tumor growth in xenograft mice}

To further investigate the role of HOXD10 in human HCC, a xenograft mouse model was employed. HOXD10 unexpressed and re-expressed SMMC7721 cells were inoculated into nude mice subcutaneously. The tumor volume in HOXD10 unexpressed and re-expressed SMMC7721 cell transplanted xenograft mice was $527.22 \pm 271.23 \mathrm{~mm}^{3}$ vs. $212.00 \pm 75.93 \mathrm{~mm}^{3}$. The tumor volume was smaller in HOXD10 re-expressed SMMC7721 cell xenograft mice compared to HOXD10 unexpressed SMMC7721 cell xenograft mice $(P<0.001$, Fig. 7a). The tumor weights were $0.17 \pm 0.09 \mathrm{~g}$ vs. $0.06 \pm 0.02 \mathrm{~g}$ $(P<0.05)$ in HOXD10 unexpressed and re-expressed SMMC7721 cell xenografts. The tumor weight was significantly reduced after re-expression of HOXD10 $(P<0.05$, Fig. $7 b)$. The results demonstrate that HOXD10 suppresses HCC cell growth in vivo.

\section{Discussion}

HCC is a complex disease. Hepatocarcinogenesis involves hepatocyte injury, inflammation, proliferation, and genomic instability, which lead to alteration in several oncogenic pathways, including EGFR, AKT, WNT, and ERK signaling [25-28]. Four clusters and 39 Hox genes have been identified in humans. Hox genes are conserved across species and encode transcription factors that are defined by the DNA-binding domain called the homeodomain. Hox proteins can function as monomers or homodimers to directly drive the transcription of downstream targets. The effects exerted by Hox genes are varied in different pathways with notable tissue specificity. Hox genes may serve as oncogenes or tumor suppressor genes in different cancer types [10]. The expression of HOXD10 was lost during the
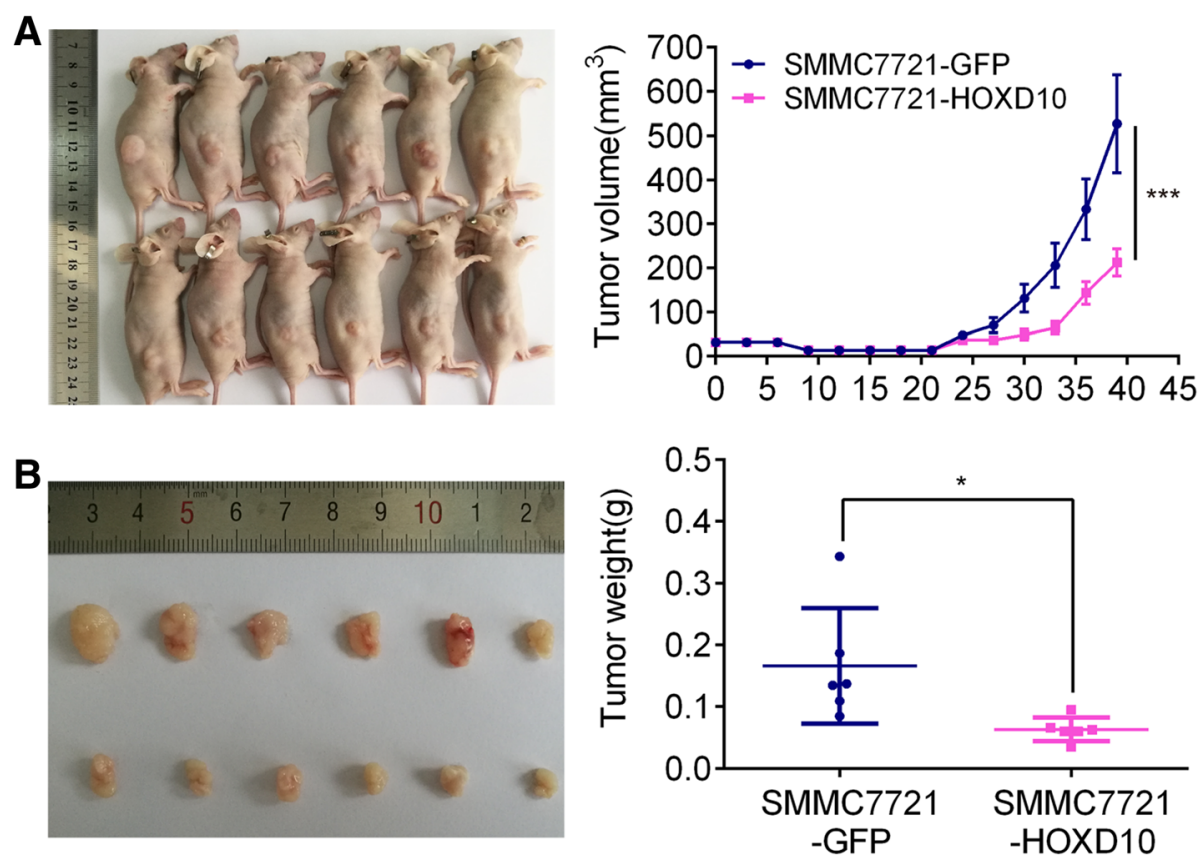

Fig. 7 HOXD10 suppresses human HCC cell tumor growth in xenograft mice. a Tumor growth curve for xenograft mice subcutaneously burdened with SMMC7721 cells stable transfected with HOXD10 or GFP control. ${ }^{* * *}<0.001$. b Tumor weights of nude mice 39 days after inoculation with SMMC7721 cells in which HOXD10 is re-expressed or unexpressed. ${ }^{*} P<0.05$ 
malignant progression of breast cancer, while the expression of HOXD10 was increased in human head and neck cancer $[29,30]$. The expression and regulation of HOXD10 in human HCC remains unclear.

In this study, we demonstrated that the expression of HOXD10 is reduced/lost frequently in HCC, and the expression of HOXD10 is regulated by promoter region methylation. HOXD10 methylation was associated with vessel cancerous embolus, tumor cell differentiation, and the 3-year survival rate. The results suggest that HOXD10 methylation may serve as a poor prognostic marker of HCC. Following up of this cohort, we only obtained 3-year OS data. Analyzing by Cox proportional hazards model, HOXD10 methylation was not an independent prognostic marker for 3-year OS after adjusting for tumor differentiation, vessel cancerous embolus, and TNM stage. Increasing the cohort number is necessary in our future study. To further clarify the function of HOXD10 in HCC, we analyzed the effects of HOXD10 on cell proliferation, apoptosis, cell cycle, cell invasion, and migration in HCC cells. In our study, HOXD10 suppressed HCC cell proliferation, induced apoptosis, and G2/M phase arrest and inhibited cell invasion and migration. Li et al. found that miR-224 directly targeted HOXD10, which triggered the down-stream p-PAK4/ MMP-9 signaling pathway, subsequently contributing to the regulation of cell migration and invasion [31]. Our study found that HOXD10 methylation is associated with vessel cancerous embolus and HOXD10 suppresses HCC cell invasion and migration. These results suggest that HOXD10 is a tumor suppressor in human HCC. The role of HOXD10 in HCC was validated by xenograft mice model in vivo.

HOXD10 targeted the IGFBP3 gene promoter region and upregulated its expression in gastric cancer [22]. Therefore, we analyzed the expression of IGFBP3 in HCC cells by western blot. The expression of IGFBP3 was upregulated by HOXD10. IGFBP3 was previously reported to interact with and inactivate ERK1/2 by inhibiting ERK1/2 phosphorylation in human non-small cell lung cancer [24]. We analyzed the effects of HOXD10 on the ERK pathway in HCC. The levels of phosphorylated ERK1/2 were reduced by HOXD10. These results were validated using the siRNA knockdown technique.

\section{Conclusion}

HOXD10 is frequently methylated in human HCC, and the expression of HOXD10 is regulated by promoter region methylation. Methylation of HOXD10 was associated with vessel cancerous embolus, tumor cell differentiation, and the 3-year survival rate in human HCC. HOXD10 suppresses HCC cell growth both in vitro and in vivo. HOXD10 suppresses human HCC by inhibiting ERK signaling.

\section{Additional file}

Additional file 1: Table S1. Primer sequences. (DOCX 13 kb)

\begin{abstract}
Abbreviations
5-aza: 5-Aza-2'-deoxycytidine; bcl-2: B cell lymphoma-2 B; BSSQ: Bisulfite sequencing; CCND1: Cyclin D1; cdc-2: Cyclin-dependent kinase 1;

ChIP: Chromatin immunoprecipitation; EGFR: Epidermal growth factor receptor; ERK: Extracellular signal-regulated kinase; GADPH: Glyceraldehyde-3phosphate dehydrogenase; HBV: Hepatitis B virus; HCC: Hepatocellular carcinoma; HOX: Homeobox; IGFBP3: Insulin-like growth factor-binding protein 3; IHC: Immunohistochemistry; MMP2: Matrix metalloprotein 2; MMP9: Matrix metalloprotein9; MTT: 3-(4,5-Dimethylthiazol-2-yl)-2,5diphenyltetrazolium bromide; NSCLC: Nonsmall-cell lung cancer; MSP: Methylation-specific PCR; RT-PCR: Reverse transcriptase polymerase chain reaction; VEGFA: Vascular endothelial growth factor $\mathrm{A}$
\end{abstract}

\section{Acknowledgements}

We sincerely thank Xiaomo Su for managing the experimental materials.

\section{Funding}

This work was supported by the following grants: National Basic Research Program of China (973 Program No.2012CB934002), National Key research and development Programme 2016YFC1303600, National Key Scientific Instrument Special Programme of China (Grant No. 2011YQ03013405), National Science Foundation of China (NSFC Nos. 81672318, 81402345, U1604281, 8167100001), Beijing Science Foundation of China (BJSFC No. 17G10035), Beijing Natural Science Foundation (7171008), and The Science and Technology Commission of Beijing Municipality (Z151100004015209).

\section{Availability of data and materials}

The datasets analyzed during the current study available from the corresponding author on reasonable request.

\section{Authors' contributions \\ YG and YP performed the research and analyzed the data. YG and MG wrote the manuscript. MG made substantial contributions to conception and design of the study. DG, MZ, and WY helped in collecting samples. EL and FF (François Fuks) provided manuscript and experimental advices. GD supervised the study. All authors read and approved the final manuscript.}

Ethics approval and consent to participate

This study was approved by the institutional review board of the Chinese PLA General Hospital.

Consent for publication

Not applicable

Competing interests

The authors declare that they have no competing interests.

\section{Publisher's Note}

Springer Nature remains neutral with regard to jurisdictional claims in published maps and institutional affiliations.

\section{Author details}

${ }^{1}$ Department of Gastroenterology and Hepatology, Chinese PLA General Hospital, \#28 Fuxing Road, Beijing 100853, China. ${ }^{2}$ Department of General Surgery, Chinese PLA General Hospital, \#28 Fuxing Road, Beijing 100853, China. ${ }^{3}$ Medical College of NanKai University, \#94 Weijin Road, Tianjin 300071, China. ${ }^{4}$ The Hillman Cancer Center, University of Pittsburgh Cancer Institute, 5117 Centre Avenue, Suite 2.18/Research, Pittsburgh, PA 15213, USA. ${ }^{5}$ Laboratory of Cancer Epigenetics, Free University of Brussels (U.L.B.), 808 Route de Lennik, 1070 Brussels, Belgium. 
Received: 15 March 2017 Accepted: 27 September 2017

Published online: 23 October 2017

\section{References}

1. Siegel R, Naishadham D, Jemal A. Cancer statistics 2013. CA Cancer J Clin. 2013:63:11-30

2. Chen W, Zheng R, Baade PD, Zhang S, Zeng H, Bray F, et al. Cancer statistics in China, 2015. CA Cancer J Clin. 2016;66:115-32.

3. El-Serag HB. Hepatocellular carcinoma. N Engl J Med. 2011;365:1118-27.

4. Farazi PA, DePinho RA. Hepatocellular carcinoma pathogenesis: from genes to environment. Nat Rev Cancer. 2006:6:674-87.

5. Villanueva A, Newell P, Chiang DY, Friedman SL, Llovet JM. Genomics and signaling pathways in hepatocellular carcinoma. Semin Liver Dis. 2007;27:55-76

6. Vogelstein B, Papadopoulos N, Velculescu VE, Zhou S, Diaz LA Jr, Kinzle KW, et al. Cancer genome landscapes. Science. 2013;339:1546-58.

7. Guo M, Ren J, Brock MV, Herman JG, Carraway HE. Promoter methylation of $\mathrm{HIN}-1$ in the progression to esophageal squamous cancer. Epigenetics. 2008;3:336-41.

8. Guo M, Ren J, House MG, Qi Y, Brock MV, Herman JG. Accumulation of promoter methylation suggests epigenetic progression in squamous cell carcinoma of the esophagus. Clin Cancer Res. 2006:12:4515-22.

9. Revill K, Wang T, Lachenmayer A, Kojima K, Harrington A, Li J, et al. Genome-wide methylation analysis and epigenetic unmasking identify tumor suppressor genes in hepatocellular carcinoma. Gastroenterology. 2013:145:1424-35. e1421-1425

10. Shah N, Sukumar S. The Hox genes and their roles in oncogenesis. Nat Rev Cancer. 2010:10:361-71.

11. Bhatlekar S, Fields JZ, Boman BM. HOX genes and their role in the development of human cancers. J Mol Med (Berl). 2014:92:811-23.

12. Cui $Y$, Gao D, Linghu E, Zhan Q, Chen R, Brock MV, et al. Epigenetic changes and functional study of HOXA11 in human gastric cancer. Epigenomics. 2015:7:201-13.

13. Hong CS, Jeong O, Piao Z, Guo C, Jung MR, Choi C, et al. HOXB5 induces invasion and migration through direct transcriptional up-regulation of betacatenin in human gastric carcinoma. Biochem J. 2015;472:393-403.

14. Yang H, Zhou J, Mi J, Ma K, Fan Y, Ning J, et al. HOXD10 acts as a tumorsuppressive factor via inhibition of the RHOC/AKT/MAPK pathway in human cholangiocellular carcinoma. Oncol Rep. 2015;34:1681-91.

15. Wang L, Chen S, Xue M, Zhong J, Wang X, Gan L, et al. Homeobox D10 gene, a candidate tumor suppressor, is downregulated through promoter hypermethylation and associated with gastric carcinogenesis. Mol Med. 2012:18:389-400.

16. Hakami F, Darda L, Stafford P, Woll P, Lambert DW, Hunter KD. The roles of HOXD10 in the development and progression of head and neck squamous cell carcinoma (HNSCC). Br J Cancer. 2014;111:807-16.

17. Mo RJ, Lu JM, Wan YP, Hua W, Liang YX, Zhuo YJ, et al. Decreased HoxD10 expression promotes a proliferative and aggressive phenotype in prostate cancer. Curr Mol Med. 2017;17:70-8.

18. Li X, Yu J, Brock MV, Tao Q, Herman JG, Liang P. Epigenetic silencing of BCL6B inactivates p53 signaling and causes human hepatocellular carcinoma cell resist to 5-FU. Oncotarget. 2015:6:11547-60.

19. Herman JG, Graff JR, Myohanen S, Nelkin BD, Baylin SB. Methylation-specific PCR: a novel PCR assay for methylation status of CpG islands. Proc Natl Acad Sci U S A. 1996;93:9821-6.

20. Jia Y, Yang Y, Liu S, Herman JG, Lu F, Guo M. SOX17 antagonizes WNT/betacatenin signaling pathway in hepatocellular carcinoma. Epigenetics. 2010;5:743-9.

21. Yan W, Wu K, Herman JG, Brock MV, Fuks F, Yang L, et al. Epigenetic regulation of DACH1, a novel Wnt signaling component in colorectal cancer. Epigenetics. 2013;8:1373-83.

22. Xue M, Fang Y, Sun G, Zhuo W, Zhong J, Qian C, et al. IGFBP3, a transcriptional target of homeobox D10, is correlated with the prognosis of gastric cancer. PLoS One. 2013;8:e81423.

23. Shahjee HM, Bhattacharyya N. Activation of various downstream signaling molecules by IGFBP-3. J Cancer Ther. 2014;5:830-5.

24. Kim JH, Choi DS, Lee OH, Oh SH, Lippman SM, Lee HY. Antiangiogenic antitumor activities of IGFBP-3 are mediated by IGF-independent suppression of Erk1/2 activation and Egr-1-mediated transcriptional events. Blood. 2011;118:2622-31.
25. Finn RS. Development of molecularly targeted therapies in hepatocellular carcinoma: where do we go now? Clin Cancer Res. 2010;16:390-7.

26. Zhang X, Yang Y, Liu X, Herman JG, Brock MV, Licchesi JD, et al. Epigenetic regulation of the Wnt signaling inhibitor DACT2 in human hepatocellular carcinoma. Epigenetics. 2013;8:373-82.

27. Chen Y, Liu Q, Wu M, Li M, Ding H, Shan X, et al. GAB2 promotes cell proliferation by activating the ERK signaling pathway in hepatocellular carcinoma. Tumour Biol. 2016;37:11763-73.

28. Forner A, Llovet JM, Bruix J. Hepatocellular carcinoma. Lancet. 2012;379:1245-55

29. Sharpe DJ, Orr KS, Moran M, White SJ, McQuaid S, Lappin TR, et al. POU2F1 activity regulates HOXD10 and HOXD11 promoting a proliferative and invasive phenotype in head and neck cancer. Oncotarget. 2014;5:8803-15.

30. Carrio M, Arderiu G, Myers C, Boudreau NJ. Homeobox D10 induces phenotypic reversion of breast tumor cells in a three-dimensional culture model. Cancer Res. 2005;65:7177-85.

31. Li Q, Ding C, Chen C, Zhang Z, Xiao H, Xie F, et al. MiR-224 promotion of cell migration and invasion by targeting Homeobox D 10 gene in human hepatocellular carcinoma. J Gastroenterol Hepatol. 2014;29:835-42.

\section{Submit your next manuscript to BioMed Central and we will help you at every step:}

- We accept pre-submission inquiries

- Our selector tool helps you to find the most relevant journal

- We provide round the clock customer support

- Convenient online submission

- Thorough peer review

- Inclusion in PubMed and all major indexing services

- Maximum visibility for your research

Submit your manuscript at www.biomedcentral.com/submit 